Arq. Bras. Med. Vet. Zootec., v.67, n.4, p.961-968, 2015

\title{
Observações clinicocirúrgicas em fraturas e luxações vertebrais toracolombares em cães
}

\author{
[Clinical and surgical observations in thoracolumbar spinal \\ fractures and luxations in dogs] \\ B.M. Araújo ${ }^{1}$, A.C. Silva ${ }^{2}$, M.L. Figueiredo ${ }^{2}$, T.H.T. Fernandes ${ }^{2}$, D. Baraúna Junior ${ }^{2}$, \\ M.A. Bonelli ${ }^{2}$, C.C. Diogo ${ }^{2}$, M.M.A. Amorim ${ }^{2}$, E.A. Tudury ${ }^{2}$ \\ ${ }^{1}$ Aluno de pós-graduação - Universidade Federal Rural de Pernambuco - Recife, PE \\ ${ }^{2}$ Universidade Federal Rural de Pernambuco - Recife, PE
}

\begin{abstract}
RESUMO
Objetivou-se avaliar as implicações e os resultados obtidos frente aos tratamentos conservativo e cirúrgico em 37 cães sem distinção de sexo, raça ou idade, que apresentaram fraturas e luxações vertebrais (FLV) toracolombares. Cada paciente passou por anamnese, seguida por exames clínico, neurológico e radiográficos para se obter o diagnóstico neuroanatômico e a severidade da lesão. Os cães foram submetidos ao tratamento conservativo ou cirúrgico de acordo com as características de cada caso. Dos 37 animais deste estudo, 37,7\% tinham percepção de dor profunda (PDP) intacta. Vinte animais foram submetidos ao tratamento conservativo, 14 ao tratamento cirúrgico e três animais faleceram antes do tratamento. Dos animais que mantiveram a PDP intacta, a taxa de recuperação foi de $100 \%$, com tempo de recuperação total variando de sete a 75 dias, ao passo que, dos animais que perderam a PDP, nenhum recuperou a deambulação voluntária. Dos 14 animais operados, $42,8 \%$ apresentaram alterações vertebrais não visibilizadas ao exame radiográfico convencional, sendo necessária mudança na técnica cirúrgica planejada em 14,2\% desses casos. Nos animais que mantiveram a PDP, não houve diferença significante nas taxas nem no tempo de recuperação da deambulação voluntária, independentemente do tipo de tratamento. Entretanto, houve diferença altamente significante entre os graus que mantiveram a PDP intacta com os animais que perderam a percepção de dor profunda em relação à taxa de recuperação. A taxa de eutanásia foi menor que em outros trabalhos descritos na literatura. Dos animais sem PDP submetidos ao tratamento conservativo ou cirúrgico, 31,25\% readquiriram a capacidade de caminhar sem recuperar a PDP, sendo essa deambulação involuntária atribuída ao caminhar espinal.
\end{abstract}

Palavras-chave: cães, coluna vertebral, trauma medular, politraumatismo, paraplegia

\begin{abstract}
Our aim of this paper was to study the implications and results associated with conservative and surgical treatment of dogs with thoracolumbar Vertebral fractures and luxations (VFL) so that the information obtained can be used to obtain guidelines that lead to greater recovery rates. For such, 37 dogs presenting VFL were examined, without distinction of sex, breed, or age. Each patient had detailed history obtained, followed by clinical, neurologic and radiographic exams for obtaining neuroanatomic location and severity of the nervous and vertebral lesions. The dogs were submitted to conservative or surgical treatment according to each case. Deep pain perception (DPP) was intact in $37.7 \%$ of the 37 dogs in this study. Twenty dogs received conservative treatment, 14 underwent surgery and three dogs died before treatment. Of the dogs with intact $D P P, 100 \%$ recovered, with a recovery time varying between seven and 75 days, while none of the dogs that lost DPP recovered voluntary motion. Of the 14 dogs submitted to surgery, $42.8 \%$ had vertebral lesions that weren't detected using conventional radiography, which demonstrates the low sensitivity of this exam, and led to a need for a change in the planned surgical technique in $14.2 \%$ of these cases. In dogs with intact DPP there was no significant difference in time or rate of recovery of voluntary motion, regardless of the treatment method. However, there was a highly significant difference between the dogs which retained and lost DPP regarding recovery rates. The percentage of euthanasia was less than in other reports, probably due to the greater awareness and protectiveness of owners nowadays. Of the dogs without DPP treated either surgically
\end{abstract}

Recebido em 4 de abril de 2014

Aceito em 27 de fevereiro de 2015

E-mail: bmaraujo85@hotmail.com 
or conservatively, $31.25 \%$ recovered ambulation without recovery of DPP, characterizing this ambulation as involuntary, attributed to spinal walk.

Keywords: dogs, vertebral column, spinal trauma, politrauma, paraplegia

\section{INTRODUÇÃO}

Fraturas e luxações vertebrais (FLV) são causadas por destruições traumáticas ou patológicas de estruturas ósseas e de tecidos de suporte da coluna vertebral, podendo produzir compressões das estruturas neurais (Seim III, 2008). Estão entre as afecções neurológicas mais comuns e são um dos distúrbios mais graves e desafiadores, devido ao elevado risco de danos medulares permanentes (Weh e Kraus, 2012).

O diagnóstico baseia-se na anamnese, nos sinais clínicos agudos, nos resultados do exame neurológico e na obtenção de imagens da coluna vertebral e medula espinal (Araújo et al., 2009). Os sinais clínicos variam de dores na coluna a paresia/paralisia, dependendo da gravidade da lesão (Fluehmann et al., 2006; Weh e Kraus, 2012).

O tratamento conservativo consiste na administração de neuroprotetores, analgésicos e imobilização da coluna vertebral (McKee, 1990; Bruce et al., 2008). É indicado para aqueles animais que apresentarem deficits neurológicos leves, lesões estáveis, fraturas não deslocadas, com ausência ou discreta compressão medular (Araújo et al., 2009; Cabassu, 2012).

O tratamento cirúrgico objetiva descompressão da medula espinal, alinhamento do canal vertebral, estabilização da coluna vertebral e remoção de possíveis fragmentos ósseos do interior do canal vertebral (Bruce et al., 2008; Jeffery, 2010; Guiot e Allman, 2011; Cabassu, 2012; Weh e Kraus, 2012), sendo indicado para os animais que apresentarem deficits graves, lesões instáveis, fraturas deslocadas, evidências de compressão medular e aqueles que estejam piorando ante o tratamento conservativo (Araújo et al., 2009).

O prognóstico dos cães vítimas de fraturas/ luxações vertebrais depende principalmente da presença ou ausência de secção medular e da perda ou manutenção da percepção de dor profunda (PDP) (Araújo et al., 2009; Weh e Kraus, 2012). Pode-se ainda realizar a associação dos sinais radiográficos de deslocamento vertebral com a percepção dolorosa e alguns outros achados do exame neurológico.

Objetivou-se avaliar as implicações e os resultados obtidos frente aos tratamentos conservativo e cirúrgico em cães com FLV toracolombares, visando a uma melhor abordagem a esses pacientes e se obter diretrizes que levem a maiores taxas de recuperação.

\section{MATERIAL E MÉTODOS}

Para o experimento (licença $N^{o}$ 023/2012 da Comissão de Ética no Uso de Animais da Universidade Federal Rural de Pernambuco), foram utilizados 37 animais da espécie canina, durante o período entre março de 2011 e julho de 2012, sem distinção de sexo, raça ou idade, que apresentaram fraturas e luxações vertebrais toracolombares, diagnosticadas por meio de exames neurológico e radiográfico.

Após a anamnese, foi realizada avaliação clínica geral, verificando o estado geral e possíveis lesões não neurológicas concomitantes. $\mathrm{Na}$ ausência ou após o tratamento destas, foi realizado exame neurológico para se obter o diagnóstico neuroanatômico e a severidade da lesão. Em seguida, solicitaram-se exames complementares, de acordo com a necessidade de cada caso, sendo todos os dados transcritos em uma ficha de avaliação individual. Os graus de disfunção neurológica adaptados de Bruce et al. (2008), sendo os grupos I a IV formados pelos animais que mantiveram a PDP intacta e o grupo $\mathrm{V}$, pelos animais que a perderam.

Foram considerados pertencentes ao grupo de tratamento conservativo aqueles animais que apresentaram deficits neurológicos leves (graus I, II e III), lesões estáveis, fraturas não deslocadas, com ausência ou discreta compressão medular e cães em outros graus que não se apresentaram em condições clínicas de serem submetidos a um procedimento cirúrgico ou cujos proprietários não apresentavam condições financeiras de arcar com os custos do procedimento. 
Inicialmente, todos esses pacientes foram imobilizados, a fim de se evitar lesões adicionais à medula espinal, e foram medicados com meloxicam $(0,1 \mathrm{mg} / \mathrm{kg}$, via oral [VO], SID [uma vez ao dia], por sete dias) ou prednisona $(0,5 \mathrm{mg} / \mathrm{kg}, \mathrm{VO}, \mathrm{BID}$ [duas vezes ao dia], por cinco dias, em seguida, $0,5 \mathrm{mg}$ SID, por mais cinco dias), cloridrato de tramadol $(4 \mathrm{mg} / \mathrm{kg}, \mathrm{VO}$, TID [três vezes ao dia], por 5 dias) e omeprazol $(0,7 \mathrm{mg} / \mathrm{kg}, \mathrm{VO}, \mathrm{SID}$, por $10 \mathrm{dias})$, sendo ainda recomendados cuidados gerais de enfermagem, esvaziamento vesical e fisioterapia.

Foram considerados pacientes cirúrgicos aqueles com condições orgânicas de serem submetidos ao procedimento cirúrgico e anestésico, que apresentaram deficits neurológicos graves (graus III, IV e V), lesões instáveis, fraturas deslocadas, evidências de compressão medular e animais que apresentaram piora ante o tratamento conservativo.

A fim de padronizar os procedimentos cirúrgicos, estes foram realizadas pelos mesmos membros da equipe, efetuando-se descompressão, redução e estabilização vertebral (quando necessário), utilizando-se as técnicas descritas na literatura.

Durante o período entre o atendimento inicial e a realização do procedimento cirúrgico, todos os cães desse grupo receberam o protocolo clínico e medicamentoso do tratamento conservativo.

Os animais que se beneficiaram com o tratamento cirúrgico receberam, no pósoperatório, antibioticoterapia oral com cefalexina $(25 \mathrm{mg} / \mathrm{kg}$, TID, por dez dias). Receberam também analgesia por via oral com meloxicam $(0,1 \mathrm{mg} / \mathrm{kg}$, SID, durante sete dias) ou prednisona $(0,5 \mathrm{mg} / \mathrm{kg}, \mathrm{BID}$, por cinco dias e, em seguida, $0,5 \mathrm{mg}$, SID, por mais cinco dias), cloridrato de tramadol (4mg/kg, TID, por cinco dias), dimetilsulfóxido (1g/kg, a $20 \%$, por via intravenosa lenta, SID, até completar sete dias) e omeprazol $(0,7 \mathrm{mg} / \mathrm{kg}$, VO, SID, por $10 \mathrm{dias})$, sendo realizada fisioterapia ativa e passiva, de acordo com a indicação para cada caso.

As avaliações clínicas foram realizadas três, sete, 15, 30, 60, 90, 120 e 180 dias após o início do tratamento e, mediante dados coletados das fichas de avaliação individual, foram analisadas as informações referentes aos sinais clínicos, exames complementares, tipos de tratamento e resultados dos mesmos.

A princípio, foram analisados os graus de disfunção neurológica dos animais da pesquisa, antes e após o tratamento, de acordo com os parâmetros de Bruce et al. (2008). Mediante estudo dos exames complementares, foi verificada a eficiência do exame radiográfico em predizer as alterações estruturais que deveriam ser corrigidas cirurgicamente, correlacionando os achados radiográficos pré-operatórios com os achados transcirúrgicos para constatar se o exame radiográfico foi eficiente na determinação das alterações vertebrais. Também foi observado se a técnica planejada no período pré-operatório (baseada nos achados radiográficos) foi passível de execução de acordo com os achados transcirúrgicos.

Em relação ao tratamento, foram analisadas as ocorrências de complicações pós-cirúrgicas, a taxa de recuperação das funções neurológicas (recuperação total, parcial ou ausência de recuperação), a comparação entre a recuperação dos animais que mantiveram e perderam a PDP utilizando o teste não paramétrico qui-quadrado, as taxas e o tempo de recuperação entre os animais que mantiveram a PDP intacta em relação ao tratamento conservativo e cirúrgico utilizando-se o teste não paramétrico de MannWhitney, o tempo de recuperação dos animais em relação à comparação dos graus de disfunção neurológica entre si (independentemente do tratamento), por meio do teste não paramétrico de Kruskal-Wallis, além do grau de satisfação dos proprietários com o resultado do tratamento. Todos os testes foram realizados através do software graphPad InStat. versão 3.05 com nível de significância de $5 \%$.

Em relação à recuperação neurológica, utilizaram-se os graus estabelecidos por Mendes e Arias (2012).

\section{RESULTADOS E DISCUSSÃO}

Os dados da etiologia da lesão, localização segmentar, grau de disfunção neurológica, tipo de tratamento efetuado e o resultado da terapia de 37 cães com fraturas e luxações vertebrais estão expostos na Tab. 1. 
Tabela 1. Etiologia da lesão, localização segmentar, grau de disfunção neurológica, tipo de tratamento e o resultado da terapia dos 37 cães acometidos por FLV toracolombares

\begin{tabular}{|c|c|c|c|c|c|}
\hline Animal & Etiologia da lesão & $\begin{array}{l}\text { Local da } \\
\text { lesão }\end{array}$ & $\begin{array}{c}\text { Grau de } \\
\text { disfunção } \\
\text { neurológica } \\
\end{array}$ & $\begin{array}{l}\text { Tipo de } \\
\text { tratamento }\end{array}$ & Resultado da terapia \\
\hline 1 & Acidente automobilístico & $\mathrm{T}_{7}-\mathrm{T}_{8}$ & $\mathrm{~V}$ & Conservativo & Não recuperou \\
\hline 2 & Acidente automobilístico & $\mathrm{L}_{3}-\mathrm{L}_{4}$ & V & Conservativo & $\begin{array}{l}\text { Não recuperou } \\
\text { (eutanásia) }\end{array}$ \\
\hline 3 & Acidente automobilístico & $\mathrm{T}_{7}-\mathrm{T}_{8}$ & V & Conservativo & $\begin{array}{l}\text { Não recuperou } \\
\text { (eutanásia) }\end{array}$ \\
\hline 4 & Acidente automobilístico & $\mathrm{T}_{12}-\mathrm{T}_{13}$ & IV & Cirúrgico & Recuperação total \\
\hline 5 & Acidente automobilístico & $\mathrm{T}_{12}-\mathrm{T}_{13}$ & III & Conservativo & Recuperação total \\
\hline 6 & Queda & $\mathrm{T}_{1}-\mathrm{T}_{2}$ & II & Conservativo & Recuperação total \\
\hline 7 & Acidente automobilístico & $\mathrm{L}_{3}-\mathrm{L}_{4}$ & II & Conservativo & Recuperação total \\
\hline 8 & Acidente automobilístico & $\mathrm{T}_{11}-\mathrm{T}_{12}$ & III & Conservativo & Recuperação total \\
\hline 9 & Acidente automobilístico & $\mathrm{T}_{11}-\mathrm{T}_{12}$ & $\mathrm{~V}$ & Conservativo & $\begin{array}{l}\text { Não recuperou } \\
\text { (eutanásia) }\end{array}$ \\
\hline 10 & Acidente automobilístico & $\mathrm{T}_{12}-\mathrm{T}_{13}$ & V & Conservativo & Não recuperou \\
\hline 11 & Acidente automobilístico & $\mathrm{T}_{12}-\mathrm{T}_{13}$ & IV & Cirúrgico & $\begin{array}{l}\text { Recuperação parcial } \\
\text { (óbito por IRA) }\end{array}$ \\
\hline 12 & Acidente automobilístico & $\mathrm{T}_{9}-\mathrm{T}_{10}$ & III & Conservativo & Recuperação total \\
\hline 13 & Acidente automobilístico & $\mathrm{T}_{12}-\mathrm{T}_{13}$ & V & Conservativo & $\begin{array}{l}\text { Não recuperou } \\
\text { (eutanásia) }\end{array}$ \\
\hline 14 & Acidente automobilístico & $\mathrm{T}_{12}-\mathrm{T}_{13}$ & V & Cirúrgico & $\begin{array}{c}\text { Não recuperou } \\
\text { (caminhar espinal) }\end{array}$ \\
\hline 15 & Acidente automobilístico & $\mathrm{T}_{12}-\mathrm{T}_{13}$ & III & Conservativo & Recuperação total \\
\hline 16 & Queda & $\mathrm{T}_{12}-\mathrm{T}_{13}$ & V & - & $\begin{array}{l}\text { Eutanásia sem } \\
\text { tratamento }\end{array}$ \\
\hline 17 & Queda & $\mathrm{L}_{3}-\mathrm{L}_{4}$ & V & Conservativo & Não recuperou \\
\hline 18 & Acidente automobilístico & $\mathrm{T}_{13}-\mathrm{l}_{1}$ & V & - & $\begin{array}{l}\text { Óbito (ruptura de } \\
\text { vesícula urinária) }\end{array}$ \\
\hline 19 & Queda & $\mathrm{T}_{11}-\mathrm{T}_{12}$ & V & Cirúrgico & Não recuperou \\
\hline 20 & Acidente automobilístico & $\mathrm{T}_{11}-\mathrm{T}_{12}$ & IV & Cirúrgico & Recuperação total \\
\hline 21 & Acidente automobilístico & $\mathrm{L}_{2}-\mathrm{L}_{3}$ & III & Cirúrgico & Recuperação total \\
\hline 22 & Agressão de outro cão & $\mathrm{T}_{12}-\mathrm{T}_{13}$ & V & - & Óbito (trauma torácico) \\
\hline 23 & Acidente automobilístico & $\mathrm{T}_{13}-\mathrm{L}_{1}$ & V & Cirúrgico & Não recuperou \\
\hline 24 & Acidente automobilístico & $\mathrm{T}_{11}-\mathrm{T}_{12}$ & III & Conservativo & Recuperação total \\
\hline 25 & Acidente automobilístico & $\mathrm{T}_{12}-\mathrm{T}_{13}$ & $\mathrm{~V}$ & Cirúrgico & $\begin{array}{l}\text { Não recuperou } \\
\text { (caminhar espinal) }\end{array}$ \\
\hline 26 & Acidente automobilístico & $\mathrm{T}_{11}-\mathrm{T}_{12}$ & V & Conservativo & $\begin{array}{l}\text { Não recuperou } \\
\text { (caminhar espinal) }\end{array}$ \\
\hline 27 & Acidente automobilístico & $\mathrm{T}_{11}-\mathrm{T}_{12}$ & V & Cirúrgico & Não recuperou \\
\hline 28 & Acidente automobilístico & $\mathrm{T}_{9}-\mathrm{T}_{10}$ & V & Conservativo & Não recuperou \\
\hline 29 & Acidente automobilístico & $\mathrm{L}_{1}-\mathrm{L}_{2}$ & $\begin{array}{c}\text { IV } \\
\text { (dor profunda } \\
\text { apenas na } \\
\text { cauda) }\end{array}$ & Cirúrgico & Recuperação total \\
\hline 30 & Acidente automobilístico & $\mathrm{T}_{13}-\mathrm{L}_{1}$ & $\mathrm{~V}$ & Conservativo & Não recuperou \\
\hline 31 & Acidente automobilístico & $\mathrm{T}_{12}-\mathrm{T}_{13}$ & V & Cirúrgico & $\begin{array}{l}\text { Não recuperou } \\
\text { (caminhar espinal) }\end{array}$ \\
\hline 32 & Acidente automobilístico & $\mathrm{L}_{1}-\mathrm{L}_{2}$ & V & Conservativo & Não recuperou \\
\hline 33 & Acidente automobilístico & $\mathrm{L}_{3}-\mathrm{L}_{4}$ & II & Conservativo & Recuperação total \\
\hline 34 & Queda & $\mathrm{T}_{12}-\mathrm{T}_{13}$ & I & Conservativo & Recuperação total \\
\hline 35 & Acidente automobilístico & $\mathrm{T}_{11}-\mathrm{T}_{12}$ & V & Cirúrgico & $\begin{array}{l}\text { Não recuperou } \\
\text { (caminhar espinal) }\end{array}$ \\
\hline 36 & Acidente automobilístico & $\mathrm{T}_{1}-\mathrm{T}_{12}$ & V & Cirúrgico & Não recuperou \\
\hline 37 & Acidente automobilístico & $\mathrm{T}_{13}-\mathrm{L}_{1}$ & $\mathrm{~V}$ & Cirúrgico & Não recuperou \\
\hline
\end{tabular}

IRA: Insuficiência renal aguda. 
Em relação ao grau de disfunção neurológica, $14 / 37(37,8 \%)$ dos animais tinham PDP intacta, ao passo que $23 / 37$ dos animais $(62,1 \%$ ) a perderam.

Dos 37 animais, 20 foram submetidos ao tratamento conservativo, 14 ao tratamento cirúrgico e em três não foi realizado tratamento do traumatismo medular agudo propriamente dito.

Dos 20 cães submetidos ao tratamento conservativo, apenas oito (animais 5, 6, 7, 12, 15, 24,33 e 34) estavam incluídos nos requisitos descritos para tal por Araújo et al. (2009) e Cabassu (2012), ao passo que os 12 cães restantes tinham indicação cirúrgica. No entanto, não foram operados devido ao excessivo tempo desde o acidente até o atendimento neurológico (animais 1, 10, 17, 26, 28, 32), e não opção do procedimento cirúrgico pelos proprietários por motivos financeiros $(2,3,8,9,13$ e 30). Como 11 dos 12 animais que deveriam ter sido operados não se recuperaram, pode-se afirmar que o tratamento conservativo para esses animais não foi a melhor opção.

Desses animais com indicação cirúrgica, mas com tempo excessivo desde o acidente até a avaliação neurológica, todos apresentavam ausência de dor epaxial durante a manipulação. $\mathrm{Na}$ palpação epaxial, apresentavam sinais de formação de tecido fibroso e calo ósseo exuberante que estabilizou naturalmente a fratura. Ao contrário, o animal 35 apresentava lesão crônica (30 dias), sinais de formação de tecido fibroso ao redor da fratura e grave hiperpatia vertebral no local da lesão, sendo realizada laminectomia dorsal do tipo Funkquist A e rizotomia segmentar dorsal seletiva, que solucionou o quadro de dor. Dos seis animais que não foram operados por opção dos proprietários, quatro $(2,3,9$ e 13) foram eutanasiados (todos sem PDP); um animal possuía deslocamento vertebral grave e PDP intacta (animal 8), tendo recuperação total e um sem PDP (animal 30) não se recuperou. O que ocorreu com o animal $n^{\circ} 8$ ressalta que, mesmo diante de grande deslocamento vertebral e sinais neurológicos moderados, o tratamento conservativo, ainda que não indicado isoladamente, pode trazer benefício ao animal, corroborando os relatos de Jeffery (2010).
Dos 14 animais submetidos ao tratamento cirúrgico, nove não possuíam PDP, sendo que nenhum desses recuperou a deambulação voluntária (cinco adquiriram caminhar espinal). Os cinco animais restantes (4, 11, 20, 21 e 29), que possuíam PDP intacta, quatro apresentaram recuperação total e um foi a óbito por insuficiência renal aguda (animal 11), o qual já apresentava deambulação voluntária na ocasião do óbito. Esse resultado sugere que a probabilidade de recuperação está mais relacionada ao grau de disfunção neurológica do que ao tipo de tratamento, o que indica que cirurgias vertebrais em cães sem PDP deveriam ser realizadas apenas para aliviar a dor quando os proprietários não optarem pela eutanásia.

Em relação às técnicas cirúrgicas, em seis animais $(4,11,14,20,21$ e 29) foram realizadas combinações de descompressão medular e estabilização vertebral; em dois animais (19 e 35) só foi realizada técnica de descompressão medular; e em seis animais $(23,25,27,31,36$ e 37) foi realizada apenas e estabilização vertebral. Essa variação ocorreu devido à cronicidade da lesão, na gravidade do quadro clínico e na presença ou ausência de secção medular.

Devido à formação de tecido fibroso no local da lesão (13 dias de fratura no dia da cirurgia), dificultando o alinhamento perfeito do canal vertebral, no animal $\mathrm{n}^{\circ} 21$ foi realizada apenas descompressão e estabilização vertebral sem perfeita redução do foco da fratura, seguindo as recomendações de Jeffery (2010), quando menciona que a redução incompleta sem trauma adicional é preferível em relação à redução completa que ocasionará lesão neuronal adicional. Nesse animal, houve recuperação total das funções neurológicas 30 dias após o procedimento cirúrgico, confirmando o citado por esse autor, quando também mencionou que a medula é capaz de tolerar considerável deformação em forma e tamanho, desde que permaneça estática.

Em dois animais (19 e 35), não foi necessária a realização de estabilização vertebral definitiva, devido à estabilidade do foco da fratura no momento da cirurgia.

Em oito animais, foram realizadas técnicas de descompressão medular, sendo realizada a hemilaminectomia em quatro animais, 
mini-hemilaminectomia em dois animais e a laminectomia dorsal do tipo Funkquist A em dois animais. A predominância da minihemilaminectomia e hemilaminectomia foi baseada nas orientações de Weh e Kraus (2012). Nos animais em que foi realizado a laminectomia dorsal do tipo Funkquist A, em um (14) foi observada posterior falha na estabilização, ao passo que, no outro (animal 35), não foi observada instabilidade vertebral tanto no trans quanto no pós-cirúrgico. Neste último, a escolha se deu pelo melhor acesso às raízes dorsais para realização bilateral de rizotomia segmentar dorsal como tratamento da hiperpatia vertebral que sofria por fratura crônica (Tudury et al., 2012).

Em seis animais $(23,25,27,31,36$ e 37) não foi realizada descompressão medular, apenas estabilização vertebral, devido à constatação de secção medular durante a redução vertebral (todas no grau V na avaliação neurológica précirúrgica), o que aumentaria o tempo cirúrgico/anestésico e o risco de desestabilização da coluna vertebral.

Em relação à comparação dos achados radiográficos pré-cirúrgicos com os achados macroscópicos transcirúrgicos, observou-se que, dos 14 animais operados, seis $(42,8 \%)$ apresentaram alterações vertebrais não visibilizadas ao exame radiográfico convencional, sendo necessária mudança na técnica cirúrgica planejada, o que demonstra a baixa sensibilidade desse exame, concluindo que a radiografia é suficiente para o diagnóstico de FLV, mas sem permitir a visibilização de todas as alterações ósseas existentes, corroborando Araújo et al. (2013).

De acordo com Cabassu (2012), o planejamento pré-operatório é bastante reforçado com a utilização da tomografia computadorizada (TC). Considerando as limitações do exame radiográfico constatado nessa investigação, os autores desta pesquisa passaram a recomendar a realização da TC, quando disponível, em todos os casos de FLV, mesmo nos que possam ser diagnosticados apenas com radiografias convencionais.

Dos $14(37,8 \%)$ animais submetidos ao tratamento cirúrgico, foram observadas complicações pós-cirúrgicas em dois animais
(14,2\%). Em um animal (11), observou-se quebra do pino de Steinmann, utilizado na estabilização segmentar dorsal, três dias após o procedimento cirúrgico, e, no segundo animal (25), observaram-se soltura e exteriorização do Pino de Steinmann, utilizado na estabilização segmentar dorsal, acompanhado de discreta subluxação vertebral, 50 dias após o procedimento cirúrgico, provavelmente por pouco encurvamento do implante. Nesses dois animais, as complicações foram corrigidas em novo procedimento cirúrgico.

Nos animais que mantiveram a PDP intacta, a taxa de recuperação da deambulação voluntária foi de $100 \%$, com tempo de recuperação (13 total e um parcial) variando de 7 a 75 dias (com tempo médio de 39 dias), ao passo que, dos animais que perderam a PDP, nenhum $(100 \%)$ recuperou a deambulação voluntária (cinco adquiriram caminhar espinal).

De acordo com a análise estatística entre os graus no primeiro atendimento e após a terapia, observou-se que não houve diferença significante entre os graus que mantiveram a percepção de dor profunda intacta (graus I a IV) em relação ao atendimento inicial e às taxas de recuperação. No entanto, houve diferença altamente significante entre os graus que mantiveram a PDP intacta com os animais que perderam a percepção de dor profunda (grau V) em relação à taxa de recuperação; concluindo que o parâmetro de ausência ou presença de percepção de dor profunda é um confiante indicativo do prognóstico em cães com fraturas e luxações vertebrais.

O único animal que possuía PDP intacta e não se recuperou totalmente foi a óbito com 30 dias de pós-cirúrgico por cistite e insuficiência renal aguda. Entretanto, apresentava deambulação voluntária (grau II) ao óbito. Nos 23 animais sem PDP, dois animais foram a óbito por complicações associadas ao trauma (trauma torácico e ruptura de vesícula urinária). Os 16 animais sem PDP sobreviventes (76\%) foram submetidos a carrinhos ortopédicos e cuidados gerais de enfermagem, em que, mesmo diante da ausência de recuperação funcional voluntária, todos os proprietários ficaram satisfeitos com o resultado do tratamento. 
O tempo de recuperação total, independentemente do tratamento, variou de 7 a 75 dias (com tempo médio de recuperação de 39 \pm 19 dias). Em relação aos animais submetidos ao tratamento conservativo, o tempo de recuperação variou de sete a 60 dias, com tempo médio de 37,3 \pm 19 dias. Nos animais submetidos ao tratamento cirúrgico, o tempo de recuperação variou entre 24 a 75 dias, com tempo médio de recuperação de 44,2 \pm 21 dias. De acordo com a análise estatística, não houve diferença significante em relação ao tempo de recuperação entre os animais submetidos ao tratamento cirúrgico ou conservativo, nem houve diferença no tempo de recuperação ao comparar os graus II, III e IV entre si, independentemente do tratamento. No entanto, essa informação é arriscada e não confiável, pois vale salientar que os animais submetidos ao tratamento conservativo possuíam menor grau de disfunção neurológica (graus I, II e III), enquanto os animais submetidos ao tratamento cirúrgico possuíam disfunção neurológica mais grave (graus III, IV e V).

Os resultados favoráveis desta pesquisa foram superiores a outros descritos na literatura em relação aos animais que mantiveram a nocicepção intacta, cujos resultados variaram de 80 a 96\% (McKee, 1990; Selcer et al., 1991; Bruce et al., 2008; Bali et al., 2009; Mendes e Arias, 2012; Weh e Kraus, 2012). Essa alta taxa de recuperação assinala que a abordagem terapêutica aqui adotada deve ser rotineira nos cães com FLV que mantêm a PDP intacta.

Os autores desta pesquisa concordam com Weh e Kraus (2012), quando mencionam que, uma vez que a FLV ocasione perda da percepção de dor profunda, a chance de recuperação é bastante diminuída, já que nenhum animal dessa categoria neste estudo apresentou recuperação funcional. Concorda-se também com a opinião desses autores, quando afirmam que, se os proprietários dos cães não escolherem a eutanásia, a cirurgia pode ser realizada para reduzir a dor e descomprimir as estruturas neurais. $\mathrm{O}$ uso de um carrinho pode permitir que os animais tenham uma melhor qualidade de vida, mesmo quando o seu estado neurológico não melhorar.

A taxa total de eutanásia no presente estudo foi de $13,5 \%$, resultado inferior aos de outros autores descritos na literatura, que variou de
37,4\% a 58,82\% (Olby et al., 2003; Bruce et al., 2008). Desse total, em apenas um animal $(2,7 \%)$ (16) foi indicada a eutanásia sem nenhum tratamento, mais devido a outras alterações clínicas do que em relação ao prognóstico desfavorável da fratura vertebral. Quatro animais $(10,8 \%)$, contrariamente às indicações recebidas, foram eutanasiados em outras instituições, após não responderem ao tratamento efetuado.

A relativa baixa taxa de eutanásia neste estudo não condiz com a literatura - provavelmente devido a um caráter mais protecionista dos proprietários nos dias atuais e aos incentivos e opções oferecidas pelos médicos veterinários para proporcionar uma vida digna ao cão paraplégico -, pois se observa, em outros trabalhos, que uma grande quantidade de proprietários decide pela eutanásia antes ou após o tratamento (McKee, 1990; Selcer et al., 1991; Bruce et al., 2008; Bali et al., 2009; Mendes e Arias, 2012).

Nesta pesquisa, dos 16 animais sem percepção de dor profunda sobreviventes e que foram submetidos ao tratamento conservativo ou cirúrgico, cinco $(31,25 \%)$ (animais 14, 25, 2631 e 35) readquiriram a capacidade de caminhar sem recuperar a PDP, sendo essa deambulação involuntária atribuída ao caminhar espinal, com o tempo médio para o desenvolvimento do caminhar espinal de 114 dias. Frente a isso, deve-se lembrar que cães podem recuperar a capacidade de caminhar, de forma involuntária, vários meses após uma grave lesão medular, apesar da persistente ausência de PDP.

\section{CONCLUSÕES}

O parâmetro de ausência ou presença de percepção de dor profunda é um confiante indicativo do prognóstico em cães com fraturas e luxações vertebrais, pois os animais que mantiveram a nocicepção tiveram prognóstico de recuperação favorável, contrariamente àqueles que a perderam. Nos cães que mantiverem a PDP intacta, independentemente do tratamento instituído (conservativo ou cirúrgico), as taxas e o tempo médio de recuperação foram semelhantes, ressaltando que aqueles com pior grau de disfunção foram os encaminhados à cirurgia. O exame radiográfico diagnosticou FLV em $100 \%$ dos casos, mas sem permitir a visibilização de todas as alterações ósseas 
existentes. Dessa forma, recomenda-se a realização de outros exames de imagem, quando disponíveis, para determinação exata de todas as alterações ósseas existentes, mesmo nos que possam ser diagnosticados apenas com radiografias convencionais. O parâmetro isolado da perda da percepção de dor profunda não deve desencorajar a realização da terapia, pois, nos cães que não recuperaram a nocicepção, 31,25\% desenvolveram de caminhar espinal.

\section{REFERÊNCIAS}

ARAÚJO, B.M.; BAHR ARIAS, M.V.; TUDURY, E.A. Paraplegia aguda com perda da percepção de dor profunda em cães: revisão de literatura. Clinic. Vet., v.81, p.70-82, 2009.

ARAÚJO, B.M.; FIGUEIREDO, M.L.; SILVA, A.C. et al. Diagnóstico clínico e radiográfico de luxação traumática da articulação atlantooccipital em dois cães. Arq. Bras. Med. Vet. Zootec., v.65, p.133-138, 2013.

BALI M.S.; LANG J.; JAGGY A. et al. Comparative study of vertebral fractures and luxations in dogs and cats. Vet. Comp. Orthop. Traumatol., v.22, p. 47-53, 2009.

BRUCE, C.W.; BRISSON, B.A.; GYSELINCK, $\mathrm{K}$. Spinal fractures in dogs and cats: a

retrospective evaluation of 95 cases. Vet. Comp. Orthop. Traumatol., v. 21 p.280-284, 2008.

CABASSU, J. P. Treatment of thoracic and lumbar vertebral fractures. In: EUROPEAN SOCIETY OF VETERINARY ORTHOPAEDICS AND TRAUMATOLOGY ESVOT, 2012, Bologna. Proceedings... Bologna: ESVOT, 2012. p.129131.

FLUEHMANN, G.; DOHERR, M.G.; JAGGY, A. Canine neurological diseases in a referral hospital population between 1989 and 2000 in Switzerland. J. Small. Anim. Pract., v.47, p. 582587, 2006.
GUIOT, L.P.; ALLMAN, D.A. Median sternotomy and ventral stabilization using pins and polymethylmethacrylate for a comminuted T5 vertebral fracture in a Miniature Schnauzer. Vet. Comp. Orthop. Traumatol., v.24, p.76-83, 2011.

JEFFERY, N.D. Vertebral fracture and luxation in small animals. Vet. Clin. North. Am. Small. Anim. Pract., v.40, p.809-828, 2010.

MCKEE, W.M. Spinal trauma in dogs and cats: a review of 51 cases. Vet. Rec., v.126, p.285-289, 1990.

MENDES, D.S.; ARIAS, M.V.B. Traumatismo da medula espinhal em cães e gatos: estudo prospectivo de 57 casos. Pesqui. Vet. Bras., v.12, p.1304-13012, 2012.

OLBY, N.; LEVINE, J.; HARRIS, T. et al. Long-term functional outcome of dogs with severe injuries of the thoracolumbar spinal cord: 87 cases (1996-2001). J. Am. Vet. Med. Assoc., v.222, p.762-769, 2003.

SEIM III, H.B. Cirurgia da espinha toracolombar. In: FOSSUM, T.W. Cirurgia de pequenos animais. 2.ed. São Paulo: Roca, 2008. p.1266-1267.

SELCER, R.R.; BUBB, W.J.; WALKER, T.L. Management of vertebral column fractures in dogs and cats: 211 cases (1977-1985). J. Am. Vet. Med. Assoc., v.198, p.1965-1968, 1991.

TUDURY, E.A.; ARAÚJO, B.M.; SILVA, A.C. et al. Rizotomia dorsal no tratamento de hiperpatia por fratura vertebral crônica em cão: relato de caso. In: CONGRESSO BRASILEIRO DE CIRURGIA E ANESTESIOLOGIA VETERINÁRIA, 9., 2012, Florianópolis; J. Bras. Ciênc. Anim., v.5, supl., p.311, 2012

WEH, M.; KRAUS, K.H. Spinal fractures and luxations. In: TOBIAS, K.M.; JOHNSTON, S.A. Veterinary surgery small animals. Philadelphia: Elsevier/Saunders, 2012, p.487-503. 